\title{
Symptomatic hepatic metastasis of insulinoma 15 years after pancreatic resection
}

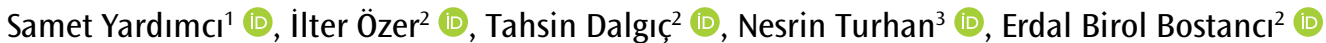

\section{ABSTRACT}

ORCID IDs of the authors:

S.Y. $0000-0003-0243-2475 ;$

i. $0.0000-0001-6902-0913$

T.D. 0000-0003-0677-2944;

N.T. $0000-0001-6566-2695$;

E.B.B. 0000-0002-0663-0156

Cite this paper as:

Yardımcı S, Özer I, Dalgı̧̧

T, Turhan N, Bostancı EB.

Symptomatic hepatic

metastasis of insulinoma

15 years after pancreatic

resection. Turk J Surg 2018

34 (3): 240-242

'Department of General Surgery, Marmara University Pendik

Training and Research Hospital,

İstanbul, Turkey

${ }^{2}$ Department of Pathology, Türkiye Yüksek Ihtisas Hospital,

Ankara, Turkey

${ }^{3}$ Department Gastroenterology Surgery, Türkiye Yüksek İhtisas

Hospital, Ankara, Turkey

\section{Corresponding Author}

Samet Yardıma

e-mail:sametyardimci@yahoo.com

Received: 23.07 .2015

Accepted: 28.09.2015

Available Online Date: 03.01.2018

c) Copyright 2018

by Turkish Surgical Association

Available online at

www.turkjsurg.com
Malignant insulinomas are infrequent endocrine tumors with miscellaneous clinical courses. In this report, we present a rare case of insulinoma that recurred with solitary hepatic metastasis 15 years after the initial resection of the primary tumor from the pancreas. A 20 -year-old woman first presented with the symptoms of hypoglycemia in 1996. The diagnosis of pancreatic insulinoma was confirmed by laboratory findings, and the initial surgery had been performed involving complete enucleation of the pancreatic mass. Histopathologically, the tumor was diagnosed as a benign adenoma. After 15 years, the patient experienced symptoms of hypoglycemia again, and laboratory findings and abdominal computed tomography identified a lesion in the liver. Intraoperative ultrasound did not show any lesion in the pancreas, and wedge resection was performed for the hepatic lesion. Postoperative blood glucose levels returned to normal. Histopathologic examination confirmed the diagnosis of liver metastasis of a neuroendocrine tumor. This is the longest interval between primary tumor and the metastasis reported in the literature, for insulinoma. Thus, it should be remembered that long-term follow-up is mandatory for the patients with insulinoma even after resection of the primary tumor. Resection is recommended when a metastatic lesion is observed.

Keywords: Insulinoma, pancreas, liver, metastasis

\section{INTRODUCTION}

Insulinomas are the most common pancreatic neuroendocrine tumors. The incidence is 3-10 cases per million individuals per year (1). Standard treatment is surgery, although accurate localization and resection of the tumor can be challenging. Five to $10 \%$ of insulinomas are malignant. Malignant insulinomas are very rare neuroendocrine tumors with varied clinical courses. After successful surgical removal, the long-term risk of recurrence is not high and for patients with a benign disease, long-term survival is expected (2). It is generally difficult to differentiate malignant and benign insulinomas. Differentiation is based on intraoperative evidence such as liver metastasis, regional lymph nodes, or local invasion, and in some patients, the metastases are diagnosed together with the recurrence of the symptoms related with hypoglycemia after surgical resection (3). In this report, we present a rare case of insulinoma that recurred with solitary hepatic metastasis 15 years after the initial pancreatic resection.

\section{CASE PRESENTATION}

A 20-year-old woman had first presented in 1996 with episodes of apathy, cloudy consciousness, syncope, and hypoglycemia. After spontaneous hypoglycemia had been confirmed by laboratory tests, a $15 \times 20 \mathrm{~mm}$ mass was detected in the pancreas by abdominal computed tomography (CT). Complete enucleation of the pancreatic mass was performed, and after surgical excision, symptoms related to hypoglycemia disappeared. There were no characteristics of multiple endocrine neoplasia syndrome either clinically or histologically. Nuclear atypia was not observed in hematoxylin and eosin (H\&E) staining, and the histopathologic diagnosis was benign adenoma.

Throughout the subsequent 15 years, patient did not show any symptoms or findings related with hypoglycemia. However, in 2011, the patient presented to our clinic with syncope and concomitant hypoglycemia. Recurrence of insulinoma was diagnosed with laboratory tests, which revealed plasma glucose, insulin, and C-peptide levels of $38 \mathrm{mg} / \mathrm{dL}, 23.2 \mathrm{mcU} / \mathrm{mL}$, and $2.71 \mathrm{ng} / \mathrm{mL}$, respectively.

After the biochemical diagnosis had been made, abdominal CT was conducted. Abdominal CT scan could not show any pancreatic mass; however, it identified a $12.5-\mathrm{mm}$ hypervascular lesion in the segment 8 of the liver (Figure 1) and a 24-mm liver hemangioma in the segment 7. A somatostatin-labelled isotope scan did not show any marked uptake of the radiopharmaceutical by the liver or the pancreas. Endoscopic ultrasound was performed for detecting a possible recurrent insulinoma but did not show any suspected lesion suggesting insulinoma. Resection of the hepatic lesion was planned. Verbal and written informed consent was obtained from the patient for both surgery and potential medical reports before the operation. 


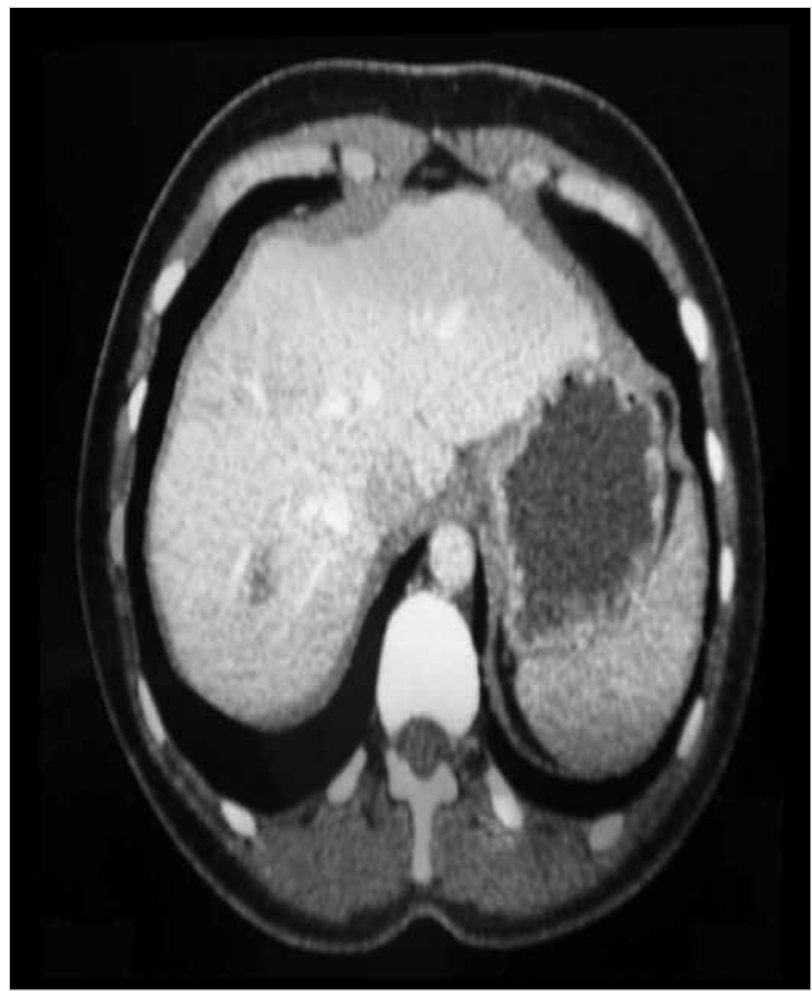

Figure 1. Hypervascular lesion in the segment 8 of the liver
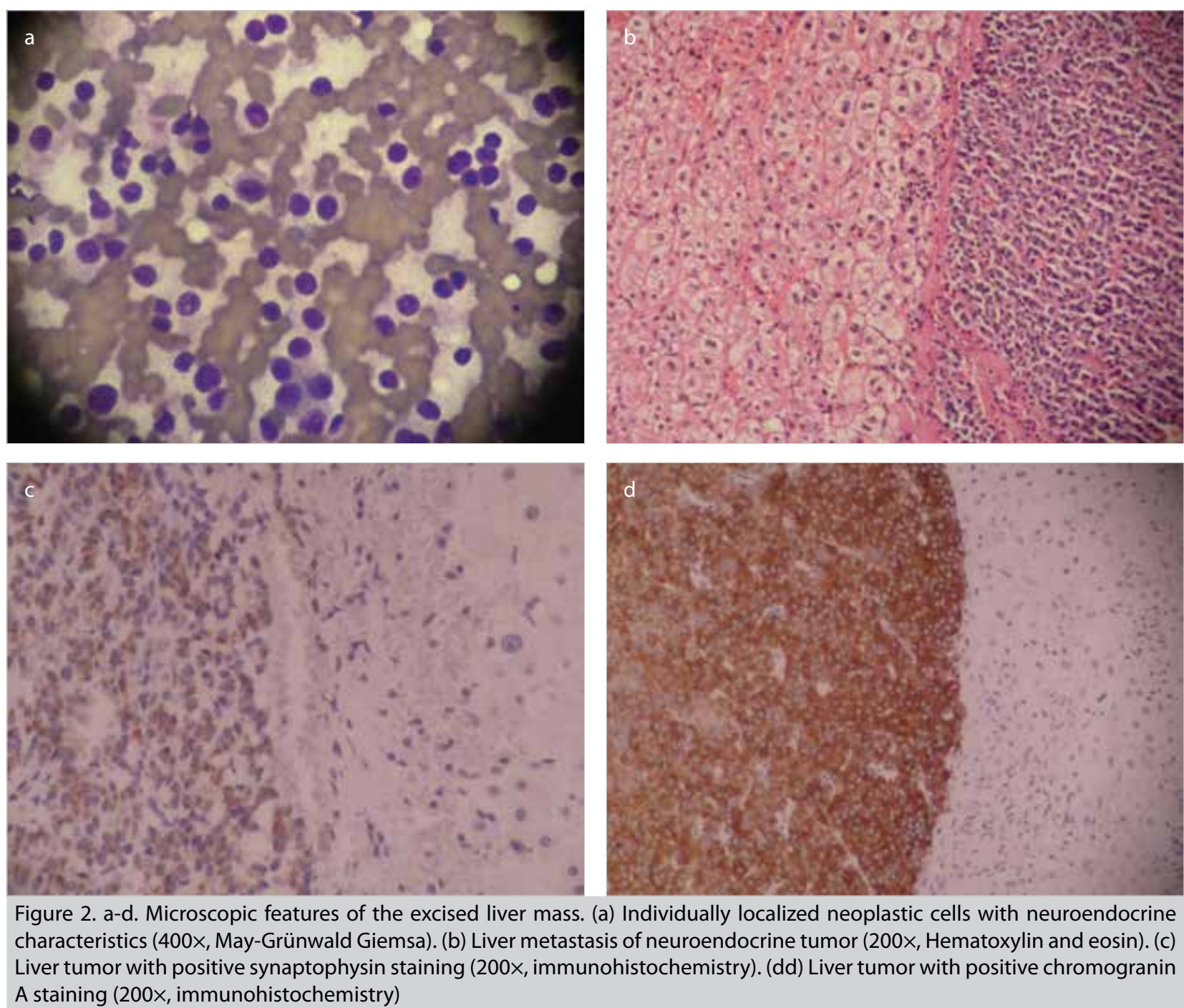

Figure 2. a-d. Microscopic features of the excised liver mass. (a) Individually localized neoplastic cells with neuroendocrine characteristics (400x, May-Grünwald Giemsa). (b) Liver metastasis of neuroendocrine tumor (200x, Hematoxylin and eosin). (c) Liver tumor with positive synaptophysin staining $(200 \times$, immunohistochemistry). (dd) Liver tumor with positive chromogranin A staining (200x, immunohistochemistry)
Intraoperative ultrasound was performed and did not show any suspected lesion in the pancreas. Wedge resection of the hepatic lesion was performed. Postoperative blood glucose levels were returned to normal and no symptoms of hypoglycemia were seen. Histopathologic examination confirmed the diagnosis of neuroendocrine tumor metastasis. The tumor was positive for synaptophysin and chromogranin A immunostaining (Figure 2). The proliferative rate, measured by $\mathrm{KI}-67$ index was $1.6 \%$. Surgical margins were free of tumor. The patient was discharged on postoperative day 6 without any complication. The patient has been asymptomatic for subsequent 4 years.

\section{DISCUSSION}

Approximately $80 \%$ of insulinomas are single benign lesions, $10 \%$ are malignant tumors, and the remaining $10 \%$ are multiple benign tumors (4). Patients with benign insulinomas generally have good prognosis if complete resection of the primary tumor can be performed. Even some patients with malignant insulinomas may survive for long periods due to the slow growth characteristic (2). The time interval between the presentation of the primary tumor and the metastasis may be long. Kobayashi et al. (5) reported a solitary non-functioning metastasis 14 years after the resection of a malignant pancreatic insulinoma. The case was diagnosed as malignant after the initial surgery. Further, Gonzalez et al. (6) reported a symptomatic hepatic metastasis 9 years after the initial pancreatic resection and the case had

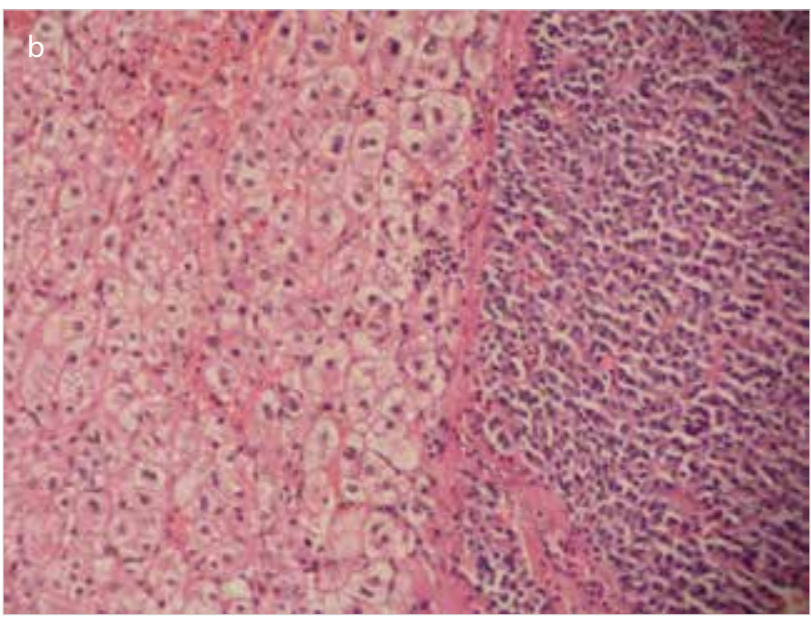


initially been considered as benign. This was the longest interval between primary tumor and the metastasis that is reported in the English literature until our report. We should remember that long-term follow-up is mandatory for these patients. Further, this case illustrates that metastasis of a pancreatic insulinoma can cause hypoglycemia, and symptomatic treatment can be achieved with the resection of the metastasis.

Liver resection is the most effective treatment of liver metastasis of neuroendocrine tumors. Other treatment options, including arterial chemo-embolization, radiofrequency ablation, radionuclide therapy, and liver transplantation, can be used for curative purposes when liver metastases are unresectable; the morbidity and mortality in patients with insulinoma are predominantly the result of the persistent hypoglycemia rather than wide-spread dissemination of the tumor (7). Thus, palliative debulking of tumors might improve survival (8). Unfortunately, however, curative cytoreduction is possible in less than $10 \%$ of all patients with metastatic insulinomas (9). The patients who have multiple unresectable metastases in the liver can be treated with chemotherapy via a catheter introduced in the hepatic artery (10). This patient had a solitary resectable lesion in the liver and was treated with liver resection.

\section{CONCLUSION}

We reported a case of metastatic insulinoma 15 years after the resection of the primary tumor. Life-long follow-up and resection when possible are recommended for even benign, resected islet cell tumors of pancreas.

Informed Consent: Written informed consent was obtained from patient who participated in this study.

Peer-review: Externally peer-reviewed.

Author Contributions: Concept - S.Y., E.B.B.; Design - S.Y, I.Ö.; Supervision - E.B.B.; Resource - S.Y.; Materials - N.T., T.D.; Data Collection and/ or Processing - S.Y., T.D.; Analysis and/or Interpretation - I.Ö.; Literature Search - S.Y.; Writing Manuscript - S.Y., I.Ö.; Critical Reviews - E.B.B.

Conflict of Interest: The author have no conflicts of interest to declare.

Financial Disclosure: The authors declared that this study has received no financial support.

\section{REFERENCES}

1. Dadan J, Wojskowicz P, Wojskowicz A. Neuroendocrine tumors of the pancreas. Wiad Lek 2008; 61: 43-47.

2. Mehrabi A, Fischer L, Hafezi M, Dirlewanger A, Grenacher L, Diener MK, et al. A systematic review of localization, surgical treatment options, and outcome of insulinoma. Pancreas 2014; 43: 675-686. [CrossRef]

3. Jensen RT. Pancreatic neuroendocrine tumors: overview of recent advances and diagnosis. J Gastrointest Surg 2006; 10: 324326. [CrossRef]

4. Vaidakis D, Karoubalis J, Pappa T, Piaditis G, Zografos GN. Pancreatic insulinoma: current issues and trends. Hepatobiliary Pancreat Dis Int 2010; 9: 234-241.

5. Kobayashi M, Kawa S, Kobayashi M, Imai Y, Sodeyama T, Kiyosawa K. Malignant insulinoma presenting as a nonfunctioning metastatic liver tumour, 14 years after resection of the primary tumor. J Gastroenterol 1998; 33:891-894. [CrossRef]

6. Gonzalez-Gonzalez A, María Recio-Cordova J. Liver Metastases 9 Years after Removal of a Malignant Insulinoma Which Was Initially Considered Benign. JOP 2006; 7: 226-229.

7. Yaprak O, Baş KK, Yüzer Y, Tokat Y. Metastatic neuroendocrine tumor: The only liver transplant indication in metastatic tumors. Ulus Cerrahi Derg 2012; 28: 109-112. [CrossRef]

8. Oberg K. Pancreatic endocrine tumors. Semin Oncol; 2010; 37: 594-618. [CrossRef]

9. Metz DC, Jensen RT. Gastrointestinal neuroendocrine tumors: pancreatic endocrine, tumors. Gastroenterology 2008; 135: 14691492. [CrossRef]

10. Sata N, Kimura W, Kanazawa T, Muto T. Malignant insulinoma causing liver metastasis 8 years after the initial surgery: report of a case. Surg Today 1995; 25: 640-642. [CrossRef] 